Stanton and Dodd provide a useful general survey of the palaeoecological literature. But this survey is rendered somewhat inaccessible by the fact that there is no author index. The book is overlong for what it has to say, and there is an unfortunate tendency towards Haigspeak: "Using the community ... . as an information transfer unit is most effective in the Neogene"'? The book could be twothirds of its length and still be an equivalent "information transfer unit".

I suggested previously that palaeoecology has been the absent Cinderella at the modern palaeontological ball. Stanton and Dodd's book suggests that she may have some time to wait for the Good Fairy, never mind the glass slipper.

P.G. Williamson is Assistant Professor at the Museum of Comparative Zoology, Harvard University.

\title{
Spot the potential carcinogen
}

\section{Alastair Hay}

Evaluation of Short-Term Tests for Carcinogens. Progress in Mutation Research, Vol.1. Edited by Frederick J. de Serres and John Ashby. Pp.827. ISBN 0-444-00570-6. (Elsevier/North-Holland: 1981.) Df1.295, \$125. Short-Term Tests for Chemical Carcinogens. Edited by H.F. Stich and R.H.C. San. Pp.518. ISBN 3-540-90496-4. (Springer-Verlag: 1981.) DM 142, $\$ 64.60$.

CANCER caused by contact with chemicals at the workplace and in the environment is something regulatory authorities are trying to control. The task is a daunting one. New chemicals reaching the market place each year are numbered in the thousands. Yet each chemical must be tested to assess its toxicity, and in particular its potential for causing cancer. Can this be done using tests that will satisfy the regulators, but will not bankrupt industry? And, just as important, are there assays which will give an answer in days, as opposed to the two years for the standard animal carcinogenicity study?

There would seem to be an optimistic answer to both questions. There are tests which satisfy these criteria and which can be used to predict carcinogenic activity. The first of the so called "short-term tests" for carcinogens was originally developed by Dr Bruce Ames at the University of California (the Ames test). Using bacterial strains of Salmonella, Ames showed that chemicals which caused cancer in animals would cause bacteria to mutate. This ability to cause mutation, he argued, could be exploited to test for carcinogens. Thus a chemical which was mutagenic in the Salmonella test would be considered to be a potential carcinogen.

Unfortunately it is not that simple. Neither the Ames test nor the others developed since its discovery - based on the mutation of other bacterial, fungal or mammalian cells - will identify every potential carcinogen. The short-term tests are fickle. They give better results for one class of chemical than for another. In addition, many of the tests have been evaluated with the researcher knowing both the identity of the chemical being tested and that it is carcinogenic in animals.

This kind of information is not going to be available when new chemicals are being tested. In this case, the tests will have to predict which chemicals are likely to cause cancer. Until recently short-term tests had never been evaluated under conditions in which they would be used by regulatory authorities to identify problem chemicals.

In 1976, however, a start was made to remedy this situation. Evaluation of ShortTerm Tests for Carcinogens is the result of that initiative. It is the report of a unique international programme of collaboration between scientists in many countries to look at the ability of these tests to predict carcinogenicity. The venture, started by the UK Medical Research Council and Health and Safety Executive, was broadened to include scientists from ICI's Central Toxicology Laboratory and the US National Institute of Environmental Health Sciences. The US Environmental Protection Agency and the National Cancer Research Institute in Japan also made a financial contribution to the study.

Scientists in some 60 laboratories were sent 42 chemicals, 25 carcinogens and 17 non-carcinogens. The chemicals were coded and the identities were only revealed after the investigators had submitted their final results. According to the organizers, the chemicals were selected on the basis of the information available about their carcinogenicity in animals. Examples from most of the major classes of organic chemical carcinogens were chosen, as were chemicals which are either "vital to life or are considered essential to our modern lifestyle". This latter group included three chemicals assumed to be non-carcinogens such as methionine, vitamin $\mathrm{C}$ and household sugar.

Because of the design of the study, the 30 or so tests under scrutiny were being evaluated as predictors of carcinogenic activity and not just as tests to look at the mutagenic properties of chemicals. Thankfully, the results of this expensive but unique exercise in collaboration show that the tests have an important role to play in any programme to screen for potential carcinogens.
In the introductory chapters to this book the editors set out the reasons for the study; the basis for selection of chemicals used; the animal carcinogenicity data on these chemicals; and a summary of the results obtained by the laboratories. In the remaining 60 or so chapters each investigating team describes their own experimental protocol and discusses the results obtained in detail.

The book contains a wealth of material which will be of immense value to anyone interested in identifying chemicals which are potential carcinogens. The results of the study show that the most effective tests are those which use bacteria such as Salmonella or Escherichia coli. However, these bacterial tests do fail to pick out some potential carcinogens which other fungal or mammalian tests will identify. But these other tests in turn fail to identify chemicals which the bacteria pick out. All tests have false negatives and false positives and the study shows that a battery of tests will be required for satisfactory results.

The unequivocal evidence about a chemical's potential for causing cancer is always regarded as being provided by tests in animals; indeed this study was based on that premise. It is somewhat ironic, therefore, that the study has shown that animal carcinogenicity data are not always as good as they might be. Azoxybenzene is said to be a non-carcinogen in animals yet many of the in vitro tests identify it as a potential carcinogen. According to one of the editors, John Ashby, this suggests that the negative carcinogenicity data on this chemical which are available may not be reliable. It is recommended that azoxybenzene should be re-tested in animals.

Imaginative titles for books on screening tests for carcinogens are obviously in short supply. Neither of the two books reviewed here has what one might call an arresting label. But the titles do at least tell the reader what he or she is going to find. The second book, Short-Term Tests for Chemical Carcinogens, contains papers from 43 experts in the field, some of whom participated in the international study. The papers describe different tests in detail and the evaluation of some of them in individual laboratories.

The value of this second volume is in the scope it gives to authors to discuss the more neglected area of viral tests for carcinogens. And some authors address the complex issues of anticarcinogens, cocarcinogens, promoters, sensitizers and DNA repair inhibitors. The role these other factors play in the mechanism of carcinogenicity is still far from clear. However, with the research effort now being devoted to this area answers will not be long in coming. These two books show that the effort so far has been worthwhile, and that the short-term test has a vital part to play in detecting potential carcinogens.

Alastair Hay is a Lecturer in the Department of Chemical Pathology at the University of Leeds. 